\title{
On the Magnetic Penetration Depth in Superconducting Ultrathin Lead Films
}

\author{
A.P. DuRAJSki* AND R. SzCZĘ́ńniak \\ Institute of Physics, Częstochowa University of Technology, Av. Armii Krajowej 19, 42-200 Częstochowa, Poland
}

\begin{abstract}
In the present paper, we report a theoretical study of the magnetic London penetration depth in ultrathin $\mathrm{Pb}$ films consisting of five to ten monolayers. Our calculations were performed within the framework of the strongcoupling approach. We observed that for thin films, the thermodynamic parameter exhibits an oscillatory behaviour connected with a quantum size effect. Moreover, we proved that the London penetration depth of $\mathrm{Pb}$ films cannot be correctly described using the Bardeen-Cooper-Schrieffer theory of superconductivity due to the strong-coupling and retardation effects. The Eliashberg theory, used in this paper, goes beyond the BCS theory to include these effects which allows to describe the superconducting state on the quantitative level.
\end{abstract}

DOI: 10.12693/APhysPolA.131.1051

PACS/topics: 74.20.Fg, 74.25.Bt, 74.62.Fj

\section{Introduction}

Relationships between size effects and thermodynamic properties in the superconducting thin films are crucial for the potential applications of these materials in nanotechnology as well as other fields of science and engineering. Recent theoretical studies and experimental measurements show that the quantum size effects in a twodimensional ultrathin metal films appear as oscillations in many physical properties upon variation of film thickness [1-6]. For instance, in photoemission experiments combined with transport measurements reported by Guo et al. [3] and in scanning tunneling spectroscopy measurements reported by Eom et al. [1] the oscillations of critical temperature and superconducting order parameter as a function of the number of atomic monolayers have been found for ultrathin $\mathrm{Pb}$ films grown on $\mathrm{Si}(111)$ substrate. This phenomenon is directly related to the quantization of the electron wave vector resulting from the confinement of its motion in the direction perpendicular to the film [7]. According to the Bardeen-CooperSchrieffer (BCS) theory of superconductivity $[8,9], T_{c}$ depends exponentially on the density of states at the Fermi surface

$$
k_{\mathrm{B}} T_{c}=1.14 \hbar \omega_{D} \exp \left(-1 / N\left(\varepsilon_{\mathrm{F}}\right) V\right),
$$

where the density of states per spin oscillates with the film thickness $L$ in the following way: $N\left(\varepsilon_{\mathrm{F}}\right)=$ $\left(m_{e}^{\star} / \pi \hbar^{2} L\right)\left\lfloor 2 L / \lambda_{\mathrm{F}}\right\rfloor$. Moreover, $k_{\mathrm{B}}$ is the Boltzmann constant, $\omega_{D}$ is the Debye frequency, $V$ is the phononmediated attractive interaction at the Fermi surface, $m_{e}^{\star}$ is the electron effective mass, $\hbar$ is the Planck constant divided by $2 \pi, \lambda_{\mathrm{F}}$ denotes the Fermi electron wavelength, and $\left\lfloor 2 L / \lambda_{\mathrm{F}}\right\rfloor$ is the integer part of $2 L / \lambda_{\mathrm{F}}$ [10]. In the case of $\mathrm{Pb}$, the $\lambda_{\mathrm{F}}$ is nearly 4 monolayers (4 ML), so the oscillating period is $2 \mathrm{ML}[3,11]$.

\footnotetext{
*corresponding author; e-mail: adurajski@wip.pcz.pl
}

It was also shown that superconductivity remains surprisingly robust, even for films as thin as $5 \mathrm{ML}$ where $T_{c}=6.1 \mathrm{~K}[1]$. Next, together with the film thickness increase, the decrease of the quantum oscillations of $T_{c}$ is observed. This result was confirmed by Qin et al. in paper [6]. Moreover, we can notice that for $\mathrm{Pb}$ films in the ultrathin regime $(5-50 \mathrm{ML})$ the values of $T_{c}$ of around $6.2 \mathrm{~K}$ are about $15 \%$ lower than the bulk value of $7.2 \mathrm{~K}$. The growth of film thickness up to $500 \mathrm{ML}$ leads to the bulk value of $T_{c}$ and the superconducting energy gap which suggest the existence of a 2D to 3D transition thickness somewhere between 50 and 500 ML [1].

Motivated by the mentioned results, in our previous paper [4], we studied the thermodynamic properties of superconducting $\mathrm{Pb}$ films within the framework of the Eliashberg formalism. In particular, the energy gap, free energy difference between the superconducting and normal state, thermodynamic critical field and the specific heat were determined for an ultrathin regime (5-10 ML). We found an oscillatory behaviour of the above thermodynamic properties modulated by quantum size effects. Similar oscillations can also be observed in other physical systems [12-16]. Moreover, we proved that the thermodynamic properties of $\mathrm{Pb}$ films cannot be correctly described using the BCS theory of superconductivity due to the strong coupling and retardation effects.

In this paper, we investigate the temperature dependence of the London penetration depth in $\mathrm{Pb}$ films in a thickness range from $5 \mathrm{ML}$ to $10 \mathrm{ML}$. By comparison of our theoretical results with experimental measurements, we show that the numerical calculations conducted in the framework of Eliashberg formalism provide a qualitative description of the $\mathrm{Pb}$ ultrathin films.

\section{Theoretical model and computational methods}

In the framework of the Eliashberg formalism, the London penetration depth $\left(\lambda_{L}\right)$ can be calculated in the following way: 


$$
\frac{1}{e^{2} v_{\mathrm{F}}^{2} N\left(\varepsilon_{\mathrm{F}}\right) \lambda_{\mathrm{L}}^{2}(T)}=\frac{4}{3} \frac{\pi}{\beta} \sum_{n=1}^{M} \frac{\Delta_{n}^{2}}{Z_{n}\left[\omega_{n}^{2}+\Delta_{n}^{2}\right]^{3 / 2}},
$$

where $e$ is the electron charge, $v_{\mathrm{F}}$ is the Fermi velocity and $\beta=1 / k_{\mathrm{B}} T$ [17]. The superconducting order parameter is defined as: $\Delta_{n}=\phi_{n} / Z_{n}$, where the imaginary axis Eliashberg functions $\phi_{n} \equiv \phi\left(\mathrm{i} \omega_{n}\right)$ and $Z_{n} \equiv Z\left(\mathrm{i} \omega_{n}\right)$ can be written as follows [18]:

$$
\phi_{n}=\frac{\pi}{\beta} \sum_{m=-M}^{M} \frac{\lambda\left(\mathrm{i} \omega_{n}-\mathrm{i} \omega_{m}\right)-\mu^{\star} \theta\left(\omega_{c}-\left|\omega_{m}\right|\right)}{\sqrt{\omega_{m}^{2} Z_{m}^{2}+\phi_{m}^{2}}} \phi_{m}
$$

and

$$
Z_{n}=1+\frac{1}{\omega_{n}} \frac{\pi}{\beta} \sum_{m=-M}^{M} \frac{\lambda\left(\mathrm{i} \omega_{n}-\mathrm{i} \omega_{m}\right)}{\sqrt{\omega_{m}^{2} Z_{m}^{2}+\phi_{m}^{2}}} \omega_{m} Z_{m} .
$$

The Matsubara frequency is defined as $\omega_{n} \equiv$ $(\pi / \beta)(2 n-1)$ where $n=0, \pm 1, \pm 2, \ldots, \pm M$, and $M=$ 1100. The pairing kernel for the electron-phonon interaction is given by

$$
\begin{aligned}
& \lambda\left(\mathrm{i} \omega_{n}-\mathrm{i} \omega_{m}\right) \equiv \\
& \quad 2 \int_{0}^{\Omega_{\max }} d \omega \frac{\omega}{\omega^{2}+\left(\omega_{n}-\omega_{m}\right)^{2}} \alpha^{2} F(\omega) .
\end{aligned}
$$

In the framework of the Eliashberg formalism, the depairing interactions between the electrons are described by the Coulomb pseudopotential $\mu^{\star}$. Symbol $\theta$ denotes the Heaviside unit function and $\omega_{c}$ is the cut-off frequency set to ten times the maximum phonon frequency $\omega_{c}=10 \Omega_{\max }=100 \mathrm{meV}$.

We solved the Eliashberg equations in a numerical way using the self-consistent iteration methods [19], which have been extensively examined and successfully used in our previous study [20-23].

\section{Results and discussion}

The computed zeroth temperature London penetration depth and measured critical temperature [1] for $\mathrm{Pb}$ as a function of film thickness are presented in Fig. 1A. The rough oscillations of $\lambda_{\mathrm{L}}(0)$ caused by the quantum size effect can be readily observed. Moreover, we can note the saturation effect of oscillations for higher values of thickness. Similar situation is also observed for superconducting critical temperature [4] although the oscilations of $\lambda_{\mathrm{L}}(0)$ are out of phase to that of $T_{c}$ for low values of thickness $(L \in\{5,6,7\} \mathrm{ML})$.

The normalized $\lambda_{\mathrm{L}}^{-2}$ as a function of temperature is presented and compared with the BCS theory prediction in Fig. 1B. The differences observed between the BCS and the Eliashberg theory in a middle region of temperature are connected with the fact that the BCS approach, in contrast to the Eliashberg theory, does not include the strong coupling corrections and retardation effects of electron-phonon interactions. It should be noted that in the case of investigated $\mathrm{Pb}$ films consisting of five to ten atomic layers the electron-phonon coupling constant $\left(\lambda_{e p}\right)$ is above 1.5 [5] (BCS gives fairly good approxima- tion for $\left.\lambda_{e p} \leq 0.5\right)$. Due to the absence of appropriate experimental reports about the London penetration depth in ultrathin lead films, we compared our prediction with available data for superconducting lead nanowires [24] and for thicker lead films (150-400 A) [25]. As expected, the experimental results are more compatible with our calculations for thin films than with the predictions of BCS theory.
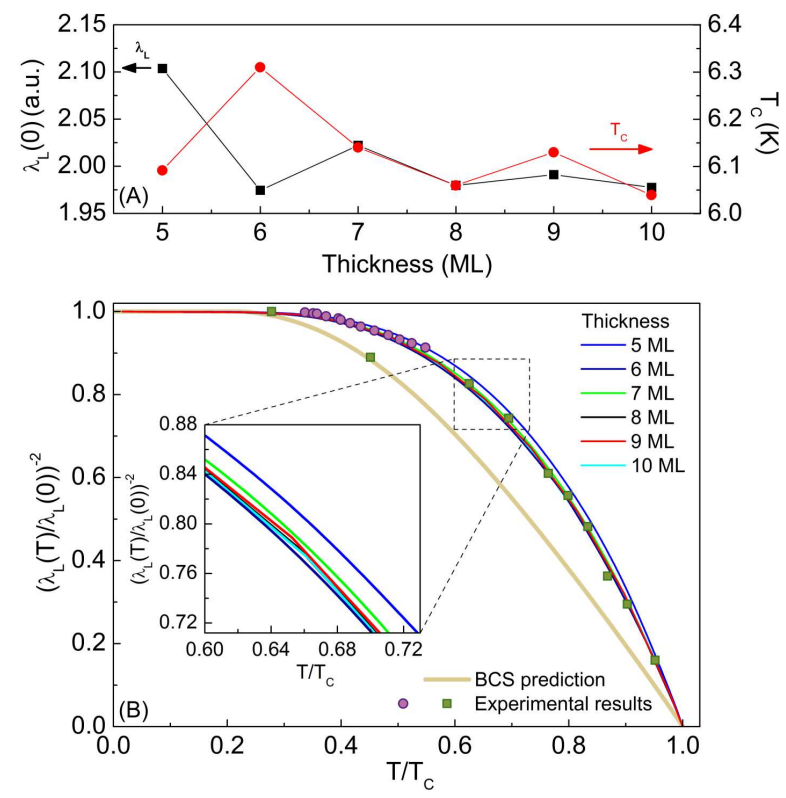

Fig. 1. (A) The zeroth temperature London penetration depth (black squares) defined as $\lambda_{\mathrm{L}}(0)=$ $e v_{\mathrm{F}} \sqrt{N\left(\varepsilon_{\mathrm{F}}\right)} \lambda_{\mathrm{L}}(T=0 \mathrm{~K})$ and critical temperature (red circles) [1] as a function of film thickness. (B) The normalized London penetration depth as a function of temperature compared with BCS prediction. The experimental results for lead nanowires (squares) and for films in the range of $150-40 \AA$ (circles) are taken from [24] and $[25]$.

\section{Conclusions}

We investigated the effects of thickness on the London penetration depth in ultrathin lead films. Calculations conducted within the framework of the strong coupling Eliashberg theory of superconductivity proves that $\lambda_{\mathrm{L}}(0)$ oscillate with film thickness. This behaviour is caused by the quantum size effects existing in the investigated range of thickness. Moreover, our study shows that the London penetration depth for strong coupling systems cannot be correctly described using the BCS theory.

We expect that the results presented in this paper can be reproduced in future measurements which can confirm our calculations. Therefore, we strongly encourage experimentalists to carry out appropriate experiments. 


\section{Acknowledgments}

A.P. Durajski acknowledges financial support from the Częstochowa University of Technology under grant No. BS/MN-203-301/2016.

\section{References}

[1] D. Eom, S. Qin, M.-Y. Chou, C.K. Shih, Phys. Rev. Lett. 96, 027005 (2006).

[2] C.M. Wei, M.Y. Chou, Phys. Rev. B 75, 195417 (2007).

[3] Y. Guo, Y.-F. Zhang, X.-Y. Bao, T.-Z. Han, Z. Tang, L.-X. Zhang, W.-G. Zhu, E.G. Wang, Q. Niu, Z.Q. Qiu, J.-F. Jia, Z.-X. Zhao, Q.-K. Xue, Science 306, 1915 (2004)

[4] A.P. Durajski, Supercond. Sci. Technol. 28, 095011 (2015).

[5] I.Y. Sklyadneva, R. Heid, K.-P. Bohnen, P.M. Echenique, E.V. Chulkov, Phys. Rev. B 87, 085440 (2013).

[6] S. Qin, J. Kim, Q. Niu, C.-K. Shih, Science 324, 1314 (2009)

[7] P. Wójcik, J. Supercond. Nov. Magn. 28, 339 (2015).

[8] J. Bardeen, L.N. Cooper, J.R. Schrieffer, Phys. Rev. 106, 162 (1957).

[9] J. Bardeen, L.N. Cooper, J.R. Schrieffer, Phys. Rev. 108, 1175 (1957).

[10] B.G. Orr, H.M. Jaeger, A.M. Goldman, Phys. Rev. Lett. 53, 2046 (1984).
[11] Y.-F. Zhang, S.-C. Li, X.-C. Ma, J.-F. Jia, Q.-K. Xue, Front. Phys. China 3, 323 (2006).

[12] J.K. Kalaga, A. Kowalewska-Kudłaszyk, W. Leoński, A. Barasiński, Phys. Rev. A 94, 032304 (2016).

[13] A. Kowalewska-Kudłaszyk, W. Leoński, T.D. Nguyen, V.C. Long, Phys. Scripta 2014, 014023 (2014).

[14] T. Blaszczyk, M. Ciesielski, Appl. Math. Comput. 257, 428 (2015)

[15] T. Blaszczyk, M. Ciesielski, M. Klimek, J. Leszczynski, Appl. Math. Comput. 218, 2480 (2011).

[16] D. Baleanu, T. Blaszczyk, J. Asad, M. Alipoure, Acta Phys. Pol. A 130, 688 (2016).

[17] J.P. Carbotte, Rev. Mod. Phys. 62, 1027 (1990).

[18] G.M. Eliashberg, Sov. Phys. JETP 11, 696 (1960).

[19] R. Szczȩśniak, Acta Phys. Pol. A 109, 179 (2006).

[20] A. Durajski, R. Szczȩśniak, L. Pietronero, Ann. Phys. (Berlin) 528, 358 (2016).

[21] M.W. Jarosik, I.A. Wrona, A.M. Duda, Solid State Commun. 219, 1 (2015).

[22] D. Szczȩśniak, R. Szczȩśniak, Acta Phys. Pol. A 127, 254 (2015)

[23] A. Durajski, Sci. Rep. 6, 38570 (2016).

[24] G. Stenuit, S. Michotte, J. Govaerts, L. Piraux, Supercond. Sci. Technol. 18, 174 (2005).

[25] E. Erlbach, R.L. Garwin, M.P. Sarachik, IBM J. Res. Dev. 4, 107 (1960). 